\title{
Blocking Probability Analysis of Cellular Systems under Dynamic Channel Allocation.
}

\author{
S. Anand, A. Sridharan, K. N. Sivarajan. \\ Department of ECE, Indian Institute of Science, \\ Bangalore- 560012 , India. \\ Ph: 91-80-309-2658, Fax: 91-80-360-0991 \\ anandecellular.ece.iisc.ernet.in
}

\begin{abstract}
Dynamic Channel allocation in Cellular Networks have become very popular off late due to the flexibility obtained by using any of the available channels in any cell as long as the Interference level is below a given threshold. In our problem we study Cellular networks under Dynamic Channel Allocation and propose a Two Dimensional Markov Chain model to analyze the performance of the system in terms of Blocking probabilities. In our analysis, we consider all the mobiles and base stations to be perfectly power controlled, i.e. each Mobile (Base station) transmits on any channel, such that the signal strength received at its corresponding Base station (Mobile) is unity irrespective of the actual position of the Mobile in the cell. We perform this analysis for a linear highway system with both Centralized and Distributed Channel Allocation. We look at low mobility systems in our problem. In practice, Digital European Cordless Telephony (DECT) is a standard to which our analysis is applicaphony (DECT) is a standard to which our analysis is applica-
ble. Keywords : Cellular Networks, Highway Systems, Dynamic Channel Allocation (DCA), Centralized, Distributed, Power Control
\end{abstract}

\section{Introduction}

Cellular Systems with Dynamic Channel Allocation (DCA) has been analyzed for blocking probability performances with Erlang $B$ approximations in each cell by Cimini et.al[2]. In that, they had analyzed DCA systems with an ad-hoc Erlang B approximation for each cell and showed the performance to be better than fixed channel allocation. Later, Moshe Sidi[3] devised an $n$ dimensional Markov model ( $n$ being the number of Channels) and arrived at product form solutions for low mobility systems. The number of states in this chain was $N^{(n+1)}$ where $N$ is the number of cells. We propose a two dimensional Markov chain for each cell with $\frac{n(n+1)}{2}$ states and solve this chain $N$ times for $N$ cells and once if all cells have uniform traffic. If we consider a Cellular Network along the corridors of an office or along a busy street, then the base stations of this network are collinear and such a system is called a Linear Highway System. We compute the blocking probability in such a system. Looking at micro cells as the future of cellular communications, we also analyze schemes for Distributed Dynamic Channel Allocation (DDCA).

The part of this research work done by A. Sridharan was funded by a research grant from Nortel Networks and the part of this research work done by $S$. Anand was funded by a research grant from DST.

\section{Organization}

The rest of the paper is organized as follows. In section III, we define the actual objective of our problem and the parameters to be minimized. Section IV gives the mathematical model for our problem and section $\mathrm{V}$ deals with the analysis of our model to obtain expressions and values for blocking and outage probabilities. In section VI, we compare the results obtained by our analytical model against computer simulations and finally, in section VII, we give our conclusions and scope for further work in this problem.

\section{Problem Definition}

A typical Highway Cellular System is as shown in figure 1. $\alpha_{1}, \alpha_{2}, \ldots, \alpha_{N}$ denote the positions of the base-stations in the highway of length $d$. The prob-

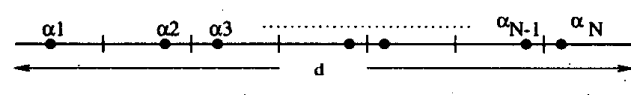

Fig. 1. A Typical Highway Cellular System.

lem is to device an analytical model for computing the blocking probability in the system under Centralized and Distributed Dynamic Channel Allocation. We are given the length of the highway $d$ and the number of cells, $N$. The Channel Allocation strategy is as follows. Each base station measures the power it receives from mobiles of all other cells at all the channels. The mobiles also measure the power they receive from all the base-stations at all the channels. The base-stations (mobiles) are power controlled. Hence the parameter the base stations and mobiles really measure is the ratio between the Interference and Signal (denoted by $\frac{I}{S}$ ). The $\frac{I}{S}$ at every channel measured by the mobiles and the basestation at every channel is compared with a threshold $\epsilon$ and a new call is alloted one of the channels that has $\frac{I}{S}<\epsilon$. We call those channels which have $\frac{I}{S}<\epsilon$ as available channels or feasible channels. The assignment of a channel among the available channels can be based on strategies like the clearest channel (the available channel which has the least $\frac{I}{S}$ ), nearest channel (the available channel with the 
largest $\frac{I}{S}$ ) and random allocation (any of the available channels at random). The performances of all the three strategies were simulated and studied by Koshy [4] and it was found that their performance is similar for large number of channels. In the analysis of the highway system, we look at the random channel allocation scheme. The difference between the admission control strategy of the Centralized and Distributed schemes is as follows.

- In the centralized scheme, a new call arriving at position $x_{i}$ in cell $i$ can be alloted a channel $k$ if the interference ${ }^{*}$ level at $x_{i}, \alpha_{i}$ and and interference levels due including the call at $\dot{x}_{i}$ at all other base stations and mobiles using channel $k$ is below the threshold. If no such channel is available, then the call at $x_{i}$ is blocked.

- In the distributed scheme, new call arriving at position $x_{i}$ in cell $i$ is alloted a channel $k$ if the interference level at $x_{i}$ and $\alpha_{i}$ are below threshold. If no such channel is available, then the call at $x_{i}$ is blocked. However, admitting the call at $x_{i}$ in channel $k$ could cause some base station $\alpha_{j}$ (with a mobile at position $x_{j}$ using channel $k$ ) in some cell $j$ or some mobile at position $x_{l}$ in cell $l$ using channel $k$ to go above threshold. In such cases, the mobile at $x_{l}$ or $x_{j}$ undergo an intra cell handoff whereby they are treated as new calls in cell $j$ or $l$ and try to find some other channel to continue the call. If no such channel is available, then the call at $x_{j}$ or $x_{l}$ is dropped.

* - Here interference level means the $\frac{I}{S}$ level. Henceforth, throughout the paper, the terms "interference level". and " $\frac{I}{S}$ level" will be used interchangeably unless specified otherwise.

\section{Mathematical Model}

\section{Given parameters}

The parameters of the highway system that are known to us are the length of the system $d$, the number of cells $N$ and the number of channels $n$.

Assumptions made in the analysis of highway cellular system with DCA

- All cells are assumed to be of equal length $\frac{d}{N}$ with the base-stations situated at the centers of each cell. - The positions of a call in any cell is uniformly distributed over the length of the cell.

- The call arrival process in any cell is a Poisson process with rate $\lambda$.

- The call holding time in any cell is Exponentially distributed with mean $\frac{1}{\mu}$.

- The signal undergoes only distance attenuation and no fading since we are looking at small cells and low mobility systems[1].
The RF signal that propagates in the air interface undergoes a short term Rayleigh fading $R$, long term $\log$ normal fading $G$ and attenuation due to the distance between the mobile and the base-station. Hence if unit power transmitted by the mobile (basestation) which is at a distance $d_{0}$ from the basestation (mobile) the power received by the basestation (mobile) $P_{r}$ is given by [5] $P_{r}=\phi R^{2} G^{2} d_{0}^{-4}$. where $\phi$ is a proportionality constant.

\section{Analysis}

In our analysis, since we are looking at the ratio of the interference power to signal power, we need to only consider the ratio of the distances of the mobiles from the base-stations and vice-versa.

Let $x_{i}$ be the random variable that denotes the position of the mobile in cell $i$. Hence, if only the mobiles in the immediate neighboring cells cause significant interference. For any two cells $M$ and $M+1$ that have calls going on at positions $x_{M}$ and $x_{M+1}$, the $\frac{I}{S}$ at the mobiles due to each other and at the base-stations $\alpha_{M}, \alpha_{M+1}$, are given by

$$
\begin{gathered}
\gamma_{B S_{M}}=\left(\frac{x_{M+1}-\alpha_{M+1}}{x_{M+1}-\alpha_{M}}\right)^{4} \\
\gamma_{B S_{M+1}}=\left(\frac{x_{M}-\alpha_{M}}{x_{M}-\alpha_{M+1}}\right)^{4} \\
\gamma_{M o b_{M}}=\left(\frac{x_{M+1}-\alpha_{M+1}}{x_{M}-\alpha_{M+1}}\right)^{4} \\
\gamma_{M o b_{M+1}}=\left(\frac{x_{M}-\alpha_{M}}{x_{M+1}-\alpha_{M}}\right)^{4}
\end{gathered}
$$

Equations $1 \ldots 4$ are obtained by taking power control into consideration. The probability that the calls at $x_{M}$ and $x_{M+1}$ can simultaneously go on at the same channel is given by $\operatorname{Pr}\left\{\left(x_{M}, x_{M+1}\right) \in \Re_{\text {feas }}\right\}$ where $\Re_{f e a s}$ is the region bounded by the lines $x_{M}+\delta x_{M+1} \geq \alpha_{M}(\theta+\delta)$

$x_{M+1}+\delta x_{M} \leq \alpha_{M+1}(\theta-\delta)$

$\phi_{M} \leq x_{M} \leq \frac{\alpha_{M}+\alpha_{M+1} \delta}{\theta+\delta}$

$\frac{\alpha_{M+1}+\alpha_{M} \delta}{\theta+\delta} \leq x_{M+1} \leq \Omega_{M}$

$\frac{(M-1) d}{N} \leq x_{M}<\frac{M d}{N}$

$\frac{M d}{N} \leq x_{M+1} \leq \frac{(M+1) d}{N}$

where $\theta=\left(\frac{2 M d}{N}\right), \phi_{M}=\max \left(\frac{(M-1) d}{N}, \frac{\alpha_{M}-\alpha_{M+1} \delta}{\theta-\delta}\right)$,

$\Omega_{M}=\min \left(\frac{\alpha_{M+1}-\alpha_{M} \delta}{\theta-\delta}, \theta\right)$ and $\delta=\epsilon^{\frac{1}{4}}$. In our analysis, we take 2 significant interferers and also model the $\frac{I}{S}$ in cell $M$ due to a mobile in cell $M+1$ to be independent if that is cell $M$ due to a mobile in cell $M-1$. Hence, if $F_{M-1}^{c}(\epsilon)$ be the probability that $\left(x_{M}, x_{M-1}\right) \notin \Re_{f e a s}$ and $F_{M+1}^{c}(\epsilon)$ be the 
probability that $\left(x_{M}, x_{M+1}\right) \notin \Re_{f e a s}$, then we have the probability that $\left(x_{M-1}, x_{M}, x_{M+1}\right) \notin \Re_{f e a s}(\epsilon)$ is given by $F_{M-1}^{c}(\epsilon) * F_{M+1}^{c}(\epsilon)$ where $*$ denotes convolution. For any channel we define the probability that a channel cannot be used due to the existence of $\Delta$ significant interferers, $p_{\text {thresh }}^{(k)}(\Delta, \epsilon)$ as

$$
p_{\text {thresh }}^{(k)}(\Delta, \epsilon)=\operatorname{Pr}\left\{X_{\Delta}^{(k)} \in \Re_{\text {feas }}^{(k)}(\Delta, \epsilon)\right\}
$$

\section{A. Centralized Channel Allocation}

For the centralized channel Channel Allocation scheme, we model the state of each cell as a 2dimensional CTMC. Each state $(m, k)$ in the CTMC denotes $m$ channels in use in a cell and $k$ channels being unusable due to violation of the interference constraint. The CTMC with its transition rates is shown in figure 2 .
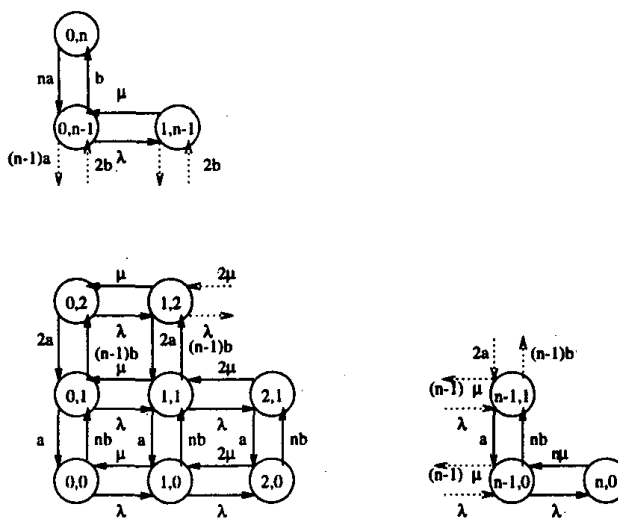

Fig. 2. State Transition Diagram for Channel Occupancy in a Cell for the Centralized Channel Allocation Scheme.

For the CTMC shown in figure 2 , the expression for the probability of occupying a given state $\pi(m, k)$ at steady state is given by [6]

$$
\pi(m . k)=\frac{1}{G}\left(\frac{\lambda}{\mu}\right)^{m} \frac{1}{m !}\left(\begin{array}{l}
n \\
k
\end{array}\right) \zeta^{k}
$$

where $G$ is a normalization term given by

$$
G=\sum_{m+k \leq n}\left(\frac{\lambda}{\mu}\right)^{m} \frac{1}{m !}\left(\begin{array}{l}
n \\
k
\end{array}\right) \zeta^{k}
$$

For computing $\zeta$, we model the process where a channel is usable or not usable as a CTMC as shown in figure 3. We define $\xi$ as the probability that a channel $k$ is usable in cell $i$. This is identical for all channels in all cells due to the frequency non-selective propagation characteristics and random channel allocation. If only the two immediate neighbors cause significant interference, and the

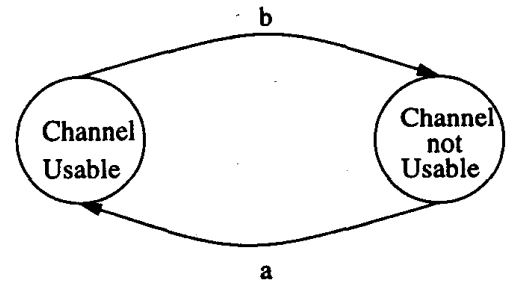

Fig. 3. CTMC model for channel interference level crossing the threshold $\epsilon$.

probability of using a channel in a cell be $p$, then, $\xi$ is given by,

$1-\xi=\sum_{\Delta=1}^{2}\left(\begin{array}{l}2 \\ \Delta\end{array}\right) p^{\Delta}(1-p)^{(2-\Delta)} p_{\text {thresh }}(\Delta, \epsilon)$

We then compute $\zeta$ as,

$$
\zeta=\frac{b}{a}=\left(\frac{1-\xi}{\xi}\right)
$$

The probability that a channel is being used in a cell, $p$ is given by

$$
p=\sum_{m+k \leq n}\left(\frac{m}{n}\right) \pi(m, k)
$$

We compute the blocking probability $P_{B}$ as

$$
P_{B}=\sum_{m+k=n} \pi(m, k)
$$

\section{B. Distributed Channel Allocation}

For the Distributed Channel Allocation Scheme, the transitions rates in a cell is modeled by a CTMC as shown in figure 4. The CTMC cannot be solved as it is, but by neglecting all the diagonal transitions in figure 4 i.e. transitions from states $(m, k)$ to $(m-1, k+1)$ and get a CTMC as in figure 2 which can be solved using equation 6 . However, the calculation of the rates $a$ and $b$ varies and hence the computation of $\zeta$. Here, we define $\Re_{f \text { eas }}$ for a cell $i$ as those call vectors $\underline{X}$ such that

$\sum_{\substack{j=i-1 \\ j \neq i}}^{i+\Delta-1}\left(\frac{x_{j}-\alpha_{j}}{x_{i}-\alpha_{j}}\right)^{4} \leq \epsilon ; \sum_{\substack{j=i-1 \\ j \neq i}}^{i+\Delta-1}\left(\frac{x_{j}-\alpha_{j}}{x_{j}-\alpha_{i}}\right)^{4} \leq \epsilon$ for $\Delta=1,2$. Again, if the probability of one interferer causing the $\frac{I}{S}$ to go above threshold is given by $F^{c}(\epsilon)$, then the probability of calls going on in both the immediate neighbors causing $\frac{I}{S}$ to go above threshold is $F^{c}(\epsilon) * F^{c}(\epsilon)$ where $*$ denotes convolution. The probability of blocking is also the probability of a handoff call blocking because no priority is being given to calls undergoing an intra-cell handoff. 

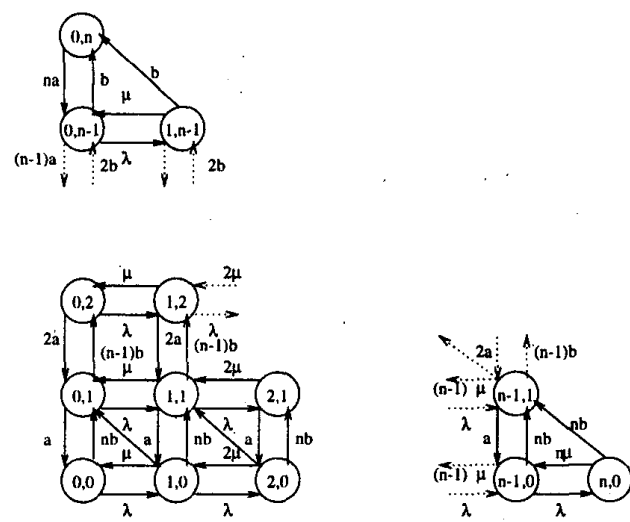

Fig. 4. State Transition Diagram for Channel Occupancy in a Cell for the Distributed Channel Allocation Scheme.

\section{Results}

We have analyzed and compared the centralized and distributed schemes for channel allocation, and compared them with simulations too. we have assumed $\mu=0.01 \mathrm{~s}^{-1}$ and have obtained simulation results for the 12 cell highway systems with both centralized and distributed channel allocation. Note from equations 6,9 and 7 , that we need to run a fixed point iteration to compute $\pi(m . k)$. The computations were done with both base station and mobile making measurements. Blocking probabilities for centralized and distributed schemes for $\rho=200$ and $\rho=300$ were analyzed and compared with computer simulations as shown in figures 5, 6,7 and 8

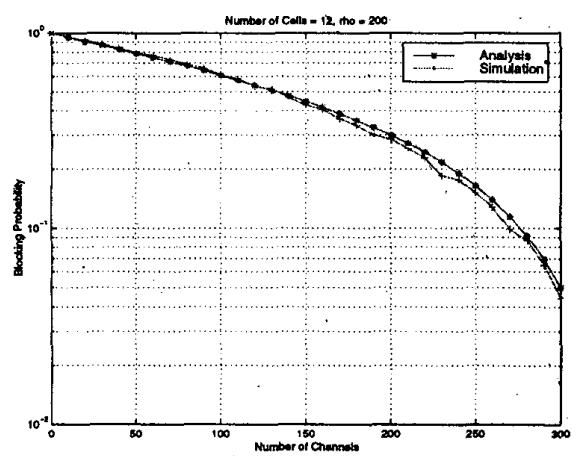

Fig. 5. Comparison of Analysis and Simulation Results for Blocking Probability in a 12 Cell Highway for $\rho=200$ and Centralized Channel Allocation.

We note that the blocking probability in the Distributed Channel Allocation scheme is one order of magnitude less than the Centralized Channel Alloca-

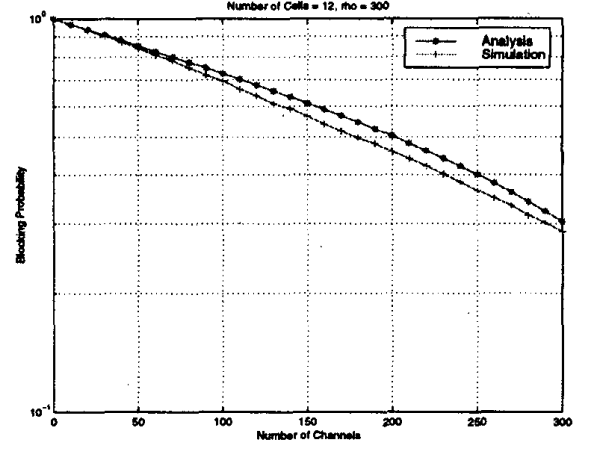

Fig. 6. Comparison of Analysis and Simulation Results for Blocking Probability in a 12 Cell Highway for $\rho=300$ and Centralized Channel Allocation.

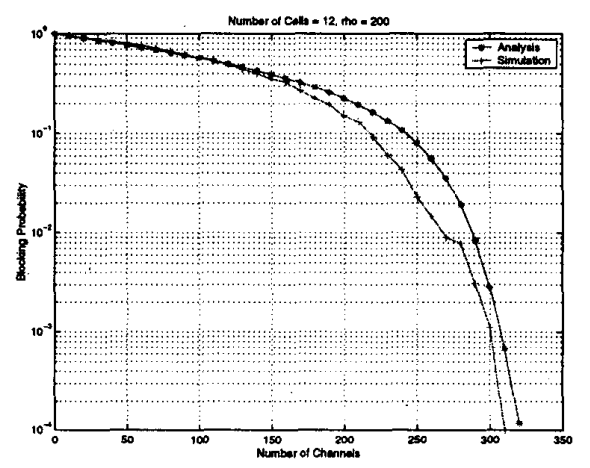

Fig. 7. Comparison of Analysis and Simulation Results for Blocking Probability in a 12 Cell Highway for $\rho=200$ and Distributed Channel Allocation.

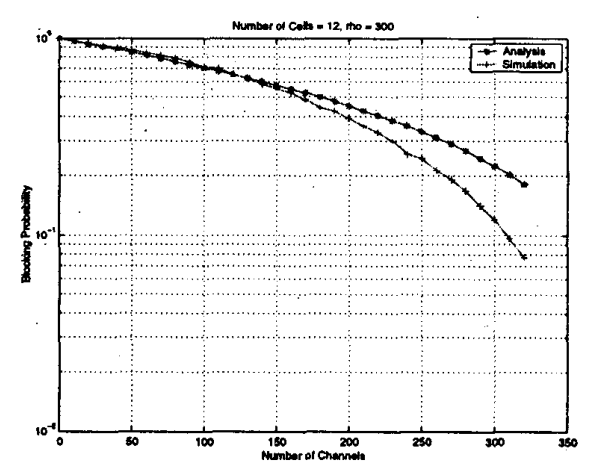

Fig. 8. Comparison of Analysis and Simulation Results for Blocking Probability in a 12 Cell Highway for $\rho=300$ and Distributed Channel Allocation. 


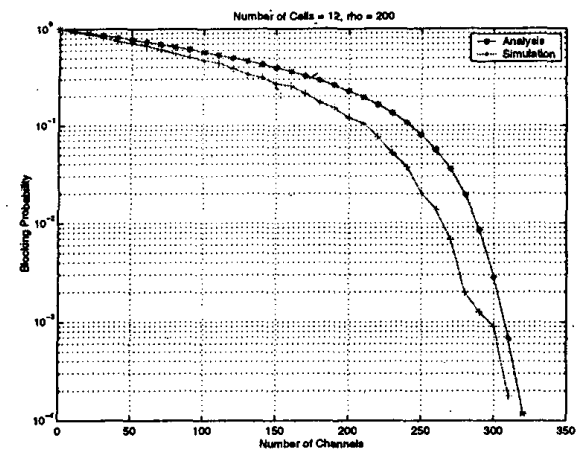

Fig. 9. Comparison of Analysis and Simulation Results for Handoff Blocking Probability in a 12 Cell Highway for $\rho=$ 200 and Distributed Channel Allocation.

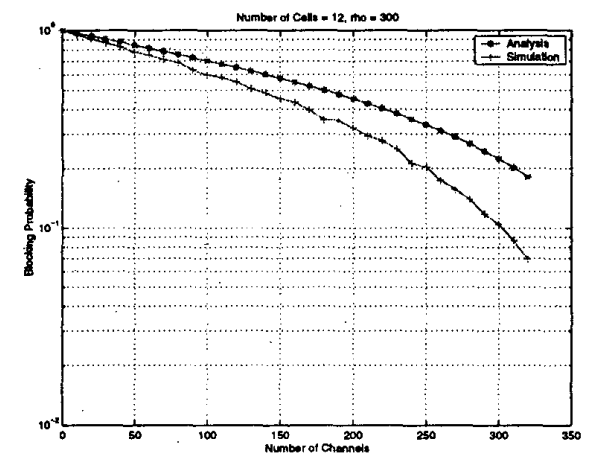

Fig. 10. Comparison of Analysis and Simulation Results for Handoff Blocking Probability in a 12 Cell Highway for $\rho$ $=300$ and Distributed Channel Allocation.

tion Scheme which also agrees with the results of $\mathrm{Ci}$ mini[2]. This is because, for the call to be accepted in the Centralized scheme $\frac{I}{S}$ at all mobiles and base stations must be below threshold whereas for the distributed scheme it is sufficient if the $\frac{I}{S}$ in the cell of call arrival alone is below threshold. However, this reduction in blocking probability is penalized in the form of call dropping. Since, in our analysis for blocking probability, we have not given any priority for already existing calls, the blocking probability is also the probability of dropping of handed off calls. This probability is also compared with results of simulations for $\rho=200$ and $\rho=300$ in figures 9 and 10 . We note from figures $5 \cdots 8$, that both for the Distributed Channel Allocation Scheme and the Centralized Channel Allocation scheme with one significant interfering neighbor, the analysis over estimates the blocking probability computed by simulations. We also note that the CTMC models are valid for two dimensional cellular systems as well but the difference occurs in computations of $\xi$ and $\zeta$.

\section{Conclusion and Future Work}

We have discussed the design of a Linear Highway System in Cellular Networks. We have devised a tool for computing the blocking probability for the linear system with distributed and centralized channel allocation scheme. We have also seen that the Distributed Channel Allocation gives much better blocking performance for the same load or conversely, we can increase the carried traffic by Distributed DCA. Though we pay a penalty due to dropping, we find that the probability of handoff calls being blocked is about $10 \%$ of the blocking probability obtained by Centralized Channel Allocation. We propose to optimize the number of cells for a specified blocking performance. This analysis is valid for the design of two dimensional cellular systems as well. We propose to extend this work for designing the optimum traffic pattern for minimizing blocking probability in a Highway System. We propose to extend our analysis for systems like GSM considering mobility and to devise Admission. Control Strategies in CDMA systems.

\section{REFERENCES}

[1] S. Mangold and M. Siebert, "Sharing Rules for DECT and PHS Providing Fair Access to the Commonly used Frequency Band." Proceedings, European Wireless (EW'99), Munich, Germany, October 1999.

[2] Leonard Cimini, Gerard Foschini, Chih-Lin I, Zoran Miljanic, "Call Blocking Performance of Distributed Algorithms for Dynamic Channel Allocation in Micro Cells", IEEE Transactions on Communications, Vol.42, No.8, 1994.

[3] M. Sidi and D. Starobinski, "New Call Blocking versus Handoff Blocking in Cellular Networks", Wireless Networks: The Journal of Mobile Communication, Computation and Information, Vol. 3, 1997.

[4] Koshy Varghese, "Decentralized Dynamic Channel Allocation in Cellular Networks." M.E Project Report, Department of ECE, Indian Institute of Science, January 1999.

[5] Theodore S. Rappaport, "Wireless Communications, Principles and Practice." Prentice Hall PTR.

[6] Keith W. Ross, "Mulitservice Loss Models in Broadband i Telecommunication Networks." Springer-Verlag. 